\title{
Canagliflozin Prevents Intrarenal Angiotensinogen Augmentation and Mitigates Kidney Injury and Hypertension in Mouse Model of Type 2 Diabetes Mellitus
}

\author{
T. Cooper Woods Ryousuke Satou Kayoko Miyata Akemi Katsurada \\ Courtney M. Dugas Natasha C. Klingenberg Vivian A. Fonseca L. Gabriel Navar
}

Departments of Physiology and of Medicine and Hypertension and Renal Center of Excellence, Tulane University

School of Medicine, New Orleans, LA, USA

\section{Keyword}

Sodium glucose cotransporter 2 inhibition · Kidney .

Angiotensinogen $\cdot$ Diabetes mellitus $\cdot$ Blood pressure

\begin{abstract}
Background: Hypertension and renal injury are common complications of type 2 diabetes mellitus (T2DM). Hyperglycemia stimulates renal proximal tubular angiotensinogen (AGT) expression via elevated oxidative stress contributing to the development of high blood pressure and diabetic nephropathy. The sodium glucose cotransporter 2 (SGLT2) in proximal tubules is responsible for the majority of glucose reabsorption by renal tubules. We tested the hypothesis that SGLT2 inhibition with canagliflozin (CANA) prevents intrarenal AGT augmentation and ameliorates kidney injury and hypertension in T2DM. Methods: We induced T2DM in New Zealand obese mice with a high fat diet (DM, 30\% fat) with control mice re-
\end{abstract}

ceiving regular fat diet (ND, 4\% fat). When DM mice exhibited $>350 \mathrm{mg} / \mathrm{dL}$ blood glucose levels, both DM- and ND-fed mice were treated with $10 \mathrm{mg} / \mathrm{kg} /$ day CANA or vehicle by oral gavage for 6 weeks. We evaluated intrarenal AGT, blood pressure, and the development of kidney injury. Results: Systolic blood pressure in DM mice $(133.9 \pm 2.0 \mathrm{~mm} \mathrm{Hg})$ was normalized by CANA $(113.9 \pm 4.0 \mathrm{~mm} \mathrm{Hg})$. CANA treatment ameliorated hyperglycemia-associated augmentation of renal AGT mRNA (148 \pm 21 copies/ng RNA in DM, and $90 \pm 16$ copies/ng RNA in DM + CANA) and protein levels as well as elevation of urinary 8-isoprostane levels. Tubular fibrosis in DM mice (3.4 \pm 0.9-fold, fibrotic score, ratio to ND) was suppressed by CANA $(0.9 \pm 0.3$-fold). Furthermore, CANA attenuated DM associated increased macrophage infiltration and cell proliferation in kidneys of DM mice. Conclusions: CANA prevents intrarenal AGT upregulation and oxidative stress and which may mitigate high blood pressure, renal tubular fibrosis, and renal inflammation in T2DM.

\section{KARGER}

(C) 2019 S. Karger AG, Basel

E-Mail karger@karger.com

www.karger.com/ajn
T. Cooper Woods, $\mathrm{PhD}$

Department of Physiology

Tulane University School of Medicine

New Orleans, LA 70112 (USA)

E-Mail twoods3@tulane.edu 


\section{Introduction}

Type 2 diabetes mellitus (T2DM) is a complex disease where hyperglycemia occurs as a result of the development of insulin resistance. In addition to the problems associated with poor glucose control, T2DM is often accompanied by high blood pressure and associated complications, including renal injury and cardiovascular disease. Excessive generation of reactive oxygen species (ROS) and activation of inflammatory responses under hyperglycemic conditions are major contributors to DM-related kidney injury and other complications $[1,2]$. The identification of targetable molecular pathways linking elevated ROS generation and the complications of T2DM requires further investigation.

Inappropriate activation of the intrarenal renin-angiotensin system (RAS), which augments intrarenal angiotensin II (Ang II) levels, appears to play a crucial role in the progression of hypertension and kidney injury $[3,4]$. A key factor in the activation of the intrarenal RAS is stimulation of renal proximal tubular angiotensinogen (AGT), which is the precursor of angiotensin peptides [3]. Elevated intrarenal AGT levels have been observed in animal models of both type- 1 and type-2 DM [5-7]. Immunohistological analyses demonstrated augmentation of AGT mainly in proximal tubules in the kidneys of DM models. Furthermore, direct treatment of rat renal proximal tubular cells (PTC) with high glucose results in elevated ROS generation and consequent stimulation of AGT production [8], indicating that high glucose-induced oxidative stress stimulates intrarenal AGT expression in DM. This high glucose-induced augmentation of proximal tubular AGT production and consequent further production of intrarenal angiotensin can facilitate the development of hypertension and nephropathy in DM.

The recent development of inhibitors of sodium glucose cotransporter 2 (SGLT2) for the treatment of T2DM has shown promise for reducing the complications of diabetes. This new class of antihyperglycemics lowers blood glucose by blocking glucose entry into proximal tubule cells through SGLT2, which is predominantly responsible for glucose reabsorption in early proximal tubules, thus leading to increased excretion of glucose in the urine. In addition to reductions in blood glucose, clinical trials of 2 SGLT2 inhibitors, canagliflozin (CANA) and dapagliflozin, have reported decreases in systolic blood pressure (SBP) of about $5 \mathrm{~mm} \mathrm{Hg}$ [9-11]. Moreover, CANA treatments improve glomerular function [12] and potentially modulate progression of albuminuria [13].

Accordingly, we hypothesized that SGLT2 inhibition of proximal tubuleglucose entrybyCANA attenuates glucose- mediated augmentation of AGT synthesis and secretion leading to decreased intrarenal angiotensin II production thus causing decreases in blood pressure and diminished renal injury in T2DM. To test this hypothesis, we investigated the effects of CANA on ROS generation and AGT expression in the kidneys of a diet-induced model of T2DM mice. In addition, we tested if CANA mitigates the development of hypertension and kidney injury in T2DM mice. Here we report that inhibition of SGLT2 reduces intrarenal AGT production coinciding with a reduction in blood pressure and mitigation of kidney inflammation and injury.

\section{Materials and Methods}

Animals and Treatments

The New Zealand Obese (NZO, NZO/HlLtJ) mouse is a polygenic model of diabetes that exhibits a higher propensity to develop hyperglycemia when fed a high fat diet $[14,15]$. NZO mice fed a high fat diet (DM, 30\% fat by weight, Teklad Diet 04059) was used to model type 2 diabetes. Control mice were fed a regular fat diet (ND, $4 \%$ fat). The diabetic state or lack thereof of both DM and ND mice was confirmed by testing the blood glucose prior to assigning mice to a treatment group. Mice were purchased from Jackson Laboratories and weaned onto the appropriate diet at 6 weeks of age. All mice were cage housed and maintained in a temperature-controlled room on a 12-h light to dark cycle, with free access to tap water and chow during acclimation. When the mice fed with high fat diet exhibited $>350 \mathrm{mg} / \mathrm{dL}$ blood glucose levels (week 0), both DM- and ND-fed mice were treated with $0.5 \%$ hydroxypropyl methylcellulose (vehicle) or $10 \mathrm{mg} / \mathrm{kg} /$ day CANA for 6 weeks by daily oral gavage.

Blood Pressure and Blood Glucose Measurements

Blood pressure of NZO mice was monitored using 2 separate methods, a tail-cuff system and a telemetry system. Initially, blood pressure measurements in all groups once a week during vehicle or CANA treatments were obtained using the tail-cuff system. Enhanced measurement of blood pressure was then obtained in a separate set using the PA-C10 radiotelemetry device (DSI) with a catheter inserted in the left carotid artery coupled with the RPC-1 receiver and software. After a recovery phase (7-14 days), mice received either vehicle or CANA treatment.

Blood glucose was measured weekly via tail snips using One Touch Ultra glucometer (Life Scan). In a subset of HFD-fed mice, blood glucose was measured continuously using the HD-XG blood glucose telemetry system (DSI). Telemetry devices were implanted in the carotid artery when mice exhibited mild hyperglycemia (250$300 \mathrm{mg} / \mathrm{dL}$ blood glucose). After a recovery phase, mice received daily vehicle treatments for 5 days followed by CANA treatments for 5 days. The treatment was performed from 10:00 to 10:30 a.m.

\section{Measurement of Body Weight and Collections of Urine and}

Tissue Samples

Body weights of mice were obtained once a week. Twenty-four hour urine samples were collected using metabolic cages once in each week. After treatment periods, mice were euthanized by conscious decapitation, and trunk blood, liver, adipose tissue and kid- 
neys were collected. Plasma samples were prepared from the collected blood immediately after collection. These samples were used for ELISAs, droplet digital PCR (ddPCR), Western blotting, and immunohistochemistry, respectively.

\section{Assays of Plasma and Urinary Components}

Plasma AGT levels were measured by an AGT ELISA (IBL). Urinary $\mathrm{Na}^{+}$and $\mathrm{K}^{+}$levels were measured using a flame photometer (Instrumentation Lab). Urinary protein levels were determined using Protein Assay Rapid Kit (Wako), and the levels were normalized by urine volume. Urinary glucose levels were determined using Infinity Glucose kit (Thermo Scientific). Urinary albumin and 8-isoprostane (a marker of renal oxidative stress) levels were measured by a Mouse Albumin ELISA Kit (Abcam) and an 8 -isoprostane EIA kit (Cayman). Urinary albumin and 8-isoprostane levels were normalized by 24 -h urine volume.

\section{Droplet Digital PCR}

ddPCR was performed to determine AGT mRNA copies in the liver, adipose tissue, and renal cortex of mice as previously described [16]. Moreover, copy numbers of renin, ACE, AT1a, and SGLT2 mRNA in renal cortex were evaluated by ddPCR. Total RNA was isolated using a commercially available RNA isolation kit (Qiagen). RNA concentration was quantified using Nanodrop 2000 (Thermo Scientific). ddPCR was performed on a Bio-Rad ddPCR system. Primers, probes, and reagents for the One-step RTddPCR system were purchased from Bio-Rad to generate cDNA and quantify gene expression. After droplet generation and PCR amplification, droplets were analyzed on the QX200 droplet reader, and target cDNA concentration was determined using the QuantaSoft analysis software (Bio-Rad). For each target gene, the amount of total RNA in a PCR reaction was determined by a pilot ddPCR, using serially diluted total RNA, in which the determined amount is in a linear range. Data are expressed as copy numbers of target gene in $1 \mathrm{ng}$ total RNA. Experimental and biologic replicates were applied.

\section{Western Blot Analysis}

AGT protein levels were determined using Western blot analysis. The Western blots were performed as previously described [17, 18]. Tissues were homogenized with $60 \mu \mathrm{L}$ lysis buffer containing $1 \%$ Triton X-100, $150 \mathrm{mmol} / \mathrm{L} \mathrm{NaCl}, 1 \mathrm{mmol} / \mathrm{L}$ EDTA, $1 \%$ Nonidet P-40, $1 \mathrm{mmol} / \mathrm{L} \mathrm{Na}_{3} \mathrm{VO}_{4}$, and $0.25 \%$ Protease Inhibitor Cocktail (Sigma). The lysates were sonicated 3 times for $10 \mathrm{~s}$ each. Total protein concentration of the supernatant was quantified using $\mathrm{Mi}$ cro BCA Protein Assay Kit (Pierce). Then, $20 \mu \mathrm{g}$ of total protein was applied to a precast NuPAGE $4-12 \%$ gel (Invitrogen). The separated proteins were transferred to a nitrocellulose membrane (Bio-Rad). After incubation of the membrane with primary and secondly antibodies, detection and analysis were performed using the Odyssey System (Li-Cor). Data were normalized based on mouse $\beta$-actin protein expression levels.

\section{Histological Analysis}

Glomerular mesangial expansion was evaluated by periodic acid-Schiff staining (PAS). Levels of renal tubule interstitial fibrosis were determined by Masson's trichrome (MT) stain. Kidney tissues were fixed in $10 \%$ buffered formalin for $24 \mathrm{~h}$, embedded in paraffin, and cut into $4 \mu \mathrm{m}$ sections. Twenty glomeruli in a kidney section were randomly selected. MT scores were obtained from 20 pictures in renal cortex for each animal. PAS and MT scores were mechanically analyzed using Image Pro-plus software to avoid bias.

\section{Immunohistochemical Staining}

To evaluate immune cell infiltration into kidneys and cell proliferation, levels of CD68 and proliferating cell nuclear antigen (PCNA) in renal cortex were analyzed by immunostaining. Formalin fixed kidney sections $(4 \mu \mathrm{m})$ were deparaffinized with xylene and dehydrated with ethanol. The samples were heated at $100{ }^{\circ} \mathrm{C}$ for $60 \mathrm{~min}$ in citrate buffer, and target proteins were visualized by using primary antibodies, a Vectastain ABC kit (VECTOR laboratories), and 3,3'-diaminobenzidine substrate kits. Samples were costained with hematoxylin before analysis. The staining was performed with an Autostainer Plus (Dako). Immunoreactivity was semi-quantitatively evaluated in a blinded test as described in histological analysis section.

\section{Statistical Analysis}

Data are expressed as means \pm SE. The data were analyzed using Student $t$ test or one-way ANOVA followed by post hoc Tukey multiple comparison test. When comparing responses over the course of treatment, where controlling for time as a covariate was desired, ANCOVA was used. A value of $p<0.05$ was considered statistically significant.

\section{Results}

\section{Effects of CANA on Hyperglycemia in T2DM Mice}

Blood glucose levels rose to $422 \pm 23 \mathrm{mg} / \mathrm{dL}$ in DM mice by week 6 compared with $148 \pm 14 \mathrm{mg} / \mathrm{dL}$ in ND mice (Fig. 1a). CANA treatment significantly reduced the blood glucose levels, and blood glucose continued to decline to a nadir of $205 \pm 24 \mathrm{mg} / \mathrm{dL}$ by week 6 . In contrast, blood glucose levels in ND mice remained unchanged $(138 \pm 7$ vs. $148 \pm 14 \mathrm{mg} / \mathrm{dL}$ in CANA vs. untreated at week 6) throughout the study and were not altered by CANA treatment.

To determine the acute effects of CANA administration, we monitored blood glucose continuously using telemetry in a separate set of DM mice. Online supplemental Figure S1a (for all online suppl. material, see www. karger.com/doi/10.1159/000499597 demonstrates a rapid, sustained, and dose-dependent reduction in blood glucose following CANA treatment. The average blood glucose during a baseline period (day 1 in online suppl. Fig. S1a) was $328 \mathrm{mg} / \mathrm{dL}$. Treatment of the mouse with $5 \mathrm{mg} / \mathrm{kg} /$ day CANA slightly lowered blood glucose levels (302 mg/dL on day 2). Ten $\mathrm{mg} / \mathrm{kg} /$ day CANA induced further reductions in blood glucose levels $(232 \mathrm{mg} / \mathrm{dL}$ on day $4,224 \mathrm{mg} / \mathrm{dL}$ on day 5, and $202 \mathrm{mg} / \mathrm{dL}$ on day 6 in online suppl. Fig. S1a). To characterize the effects of prolonged dosing, $5 \mathrm{DM}$ mice received vehicle treatment for 


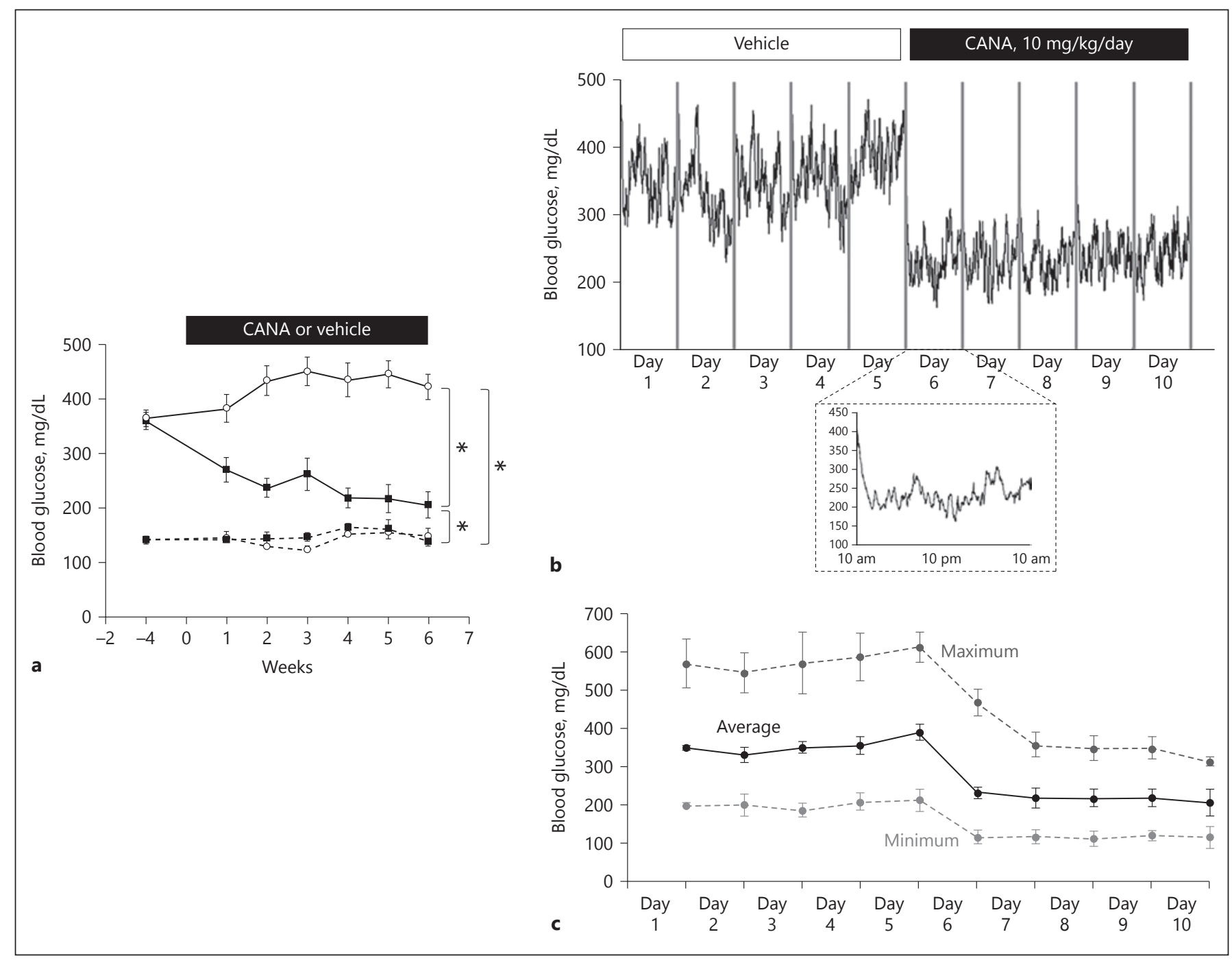

Fig. 1. Effects of CANA on hyperglycemia in T2DM mice. a Blood glucose levels measured by a glucose meter. ND + Veh: $n=12$, ND + CANA: $n=11$, DM + Veh: $n=10-12$, DM + CANA: $n=$ 14-16. Dotted lines show results in ND mice. Solid lines indicate results in DM mice. Open circles and closed squares are vehicleand CANA-treated groups, respectively. b Continuous recording of blood glucose in DM mice by a telemetry system. Average of blood glucose levels $(n=5)$ measured by a telemetry system were plotted every $1 \mathrm{~min}$. Treatments were performed from 10:00 to 10:

5 days followed by $10 \mathrm{mg} / \mathrm{kg} /$ day CANA for 5 days (Fig. 1b, c). During vehicle treatment for 5 days, blood glucose levels in the mice averaged $355 \mathrm{mg} / \mathrm{dL}$. Lower blood glucose levels during early morning compared to nighttime (active time) were observed during vehicle treatment. Blood glucose levels demonstrated a large variation over $24 \mathrm{~h}$ during the vehicle treatment (Fig. 1c, maximum minus minimum blood glucose: $370 \mathrm{mg} / \mathrm{dL}$ on
30 a.m. The top panel shows changes in blood glucose levels during vehicle treatment (day 1-5) and CANA treatment (day 6-10). The bottom panel shows changes in blood glucose levels on day 6. c Variation of blood glucose in DM mice. Average, maximum, and minimum of blood glucose levels $(n=5)$ measured by a telemetry system. Treatments were performed from 10:00 to 10:30 a.m. The panel shows changes in blood glucose levels during vehicle treatment (day 1-5) and CANA treatment (day 6-10). CANA, canagliflozin.

day 5). CANA rapidly reduced blood glucose levels within $3 \mathrm{~h}$ following treatment $(207 \pm 33.3 \mathrm{mg} / \mathrm{dL})$, and the lowered blood glucose was sustained until the next dose. The average 24- $\mathrm{h}$ blood glucose was gradually suppressed during the CANA treatment reaching $161 \pm 28 \mathrm{mg} / \mathrm{dL}$ on the 10th day of CANA treatment. Moreover, CANA reduced the variation of blood glucose (Fig. 1c, maximum minus minimum blood glucose: $199 \mathrm{mg} / \mathrm{dL}$ on day 10 ). 


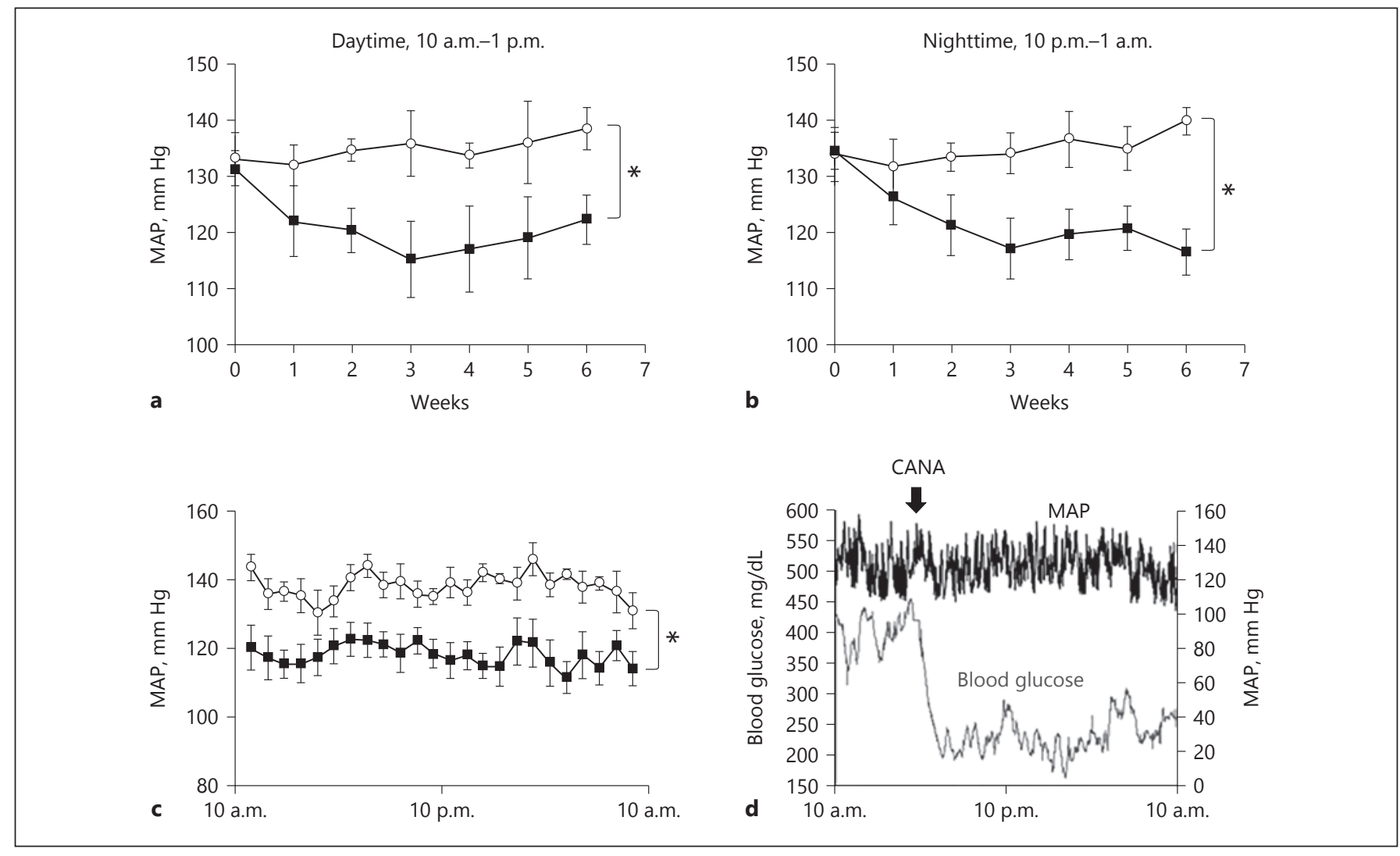

Fig. 2. Effects of CANA on blood pressure in T2DM mice. Blood pressure measured by a telemetry system. Panel (a; day time) and (b; night time) show changes in MAP. Panel (c) indicates MAP during 24 h on week 6 . d comparison between acute responses of blood glucose levels $(n=5)$ and MAP $(n=5)$ to CANA treatment. Mean \pm SE. $n=4$ in Vehicle group, $n=5$ in CANA group. Asterisk $(p<0.05)$ indicates significant difference between groups. MAP, mean arterial pressure; CANA, canagliflozin.

on week 6) and DM mice (e.g., $67 \pm 2 \mathrm{~g}$ on week 6). However, body weight gain continued in all mice treated with vehicle and CANA.

\section{Effects of CANA on Blood Pressure in T2DM Mice}

DM mice developed hypertension with increased SBP, as measured by tail-cuff plethysmography, compared to ND mice (online suppl. Fig. S3a, $118 \pm 11 \mathrm{~mm} \mathrm{Hg}$ in ND mice vs. $134 \pm 2 \mathrm{~mm} \mathrm{Hg}$ in DM mice), which supports previous finding [19]. While CANA treatment lowered SBP in DM mice on weeks 5 and $6(114 \pm 4 \mathrm{~mm} \mathrm{Hg}$ on week 6$)$, these differences were not statistically significant over the course of treatment when corrected for time as a covariate. Point by point examination of the data at weeks 5 and 6 indicated a significant reduction in blood pressure with CANA treatment. Therefore, blood pressure was measured using a telemetry system to more accurately determine the effects of CANA treatment in the DM mice (Fig. 2a-c). Significant reductions of mean arterial pres- 
sure (MAP) in response to CANA treatment were observed in both day and night periods (Fig. 2a, b, 10 a.m.-1 p.m. and 10 p.m.-1 a.m.). Continuous monitoring of blood pressure for $24 \mathrm{~h}$ (Fig. 2c) also demonstrated suppression of MAP by CANA. In addition, the telemetry monitoring revealed that elevated SBP in DM group was also attenuated by CANA (online suppl. Fig. S3b-d). Figure $2 d$ shows a comparison between acute changes in blood glucose and MAP during $24 \mathrm{~h}$ after CANA treatment. CANA rapidly reduced blood glucose levels although MAP was not altered during the initial $24 \mathrm{~h}$ demonstrating a temporal dissociation between blood glucose and MAP reductions.

\section{Effects of CANA on Urinary Sodium and Potassium}

Excretion in T2DM Mice

Urinary $\mathrm{Na}^{+}$excretion rates in $\mathrm{DM}$ mice were greater than in ND mice during week 6 (online suppl. Fig. S4a, $150 \pm 24 \mu \mathrm{mol} /$ day in ND mice vs. $282 \pm 24 \mu \mathrm{mol} /$ day in DM mice). Although CANA treatment in DM mice slightly augmented urinary $\mathrm{Na}^{+}$excretion rate $(402 \pm 37 \mu \mathrm{mol} /$ day), CANA treatment did not significantly change urinary $\mathrm{Na}^{+}$excretion rate in either ND and DM mice. DM mice exhibited higher urinary $\mathrm{K}^{+}$excretion rate during week 6 (online suppl. Fig. S4b). In both ND and DM mice, there were no significant differences in urinary $\mathrm{K}^{+}$excretion rates between vehicle-treated mice and CANA-treated mice.

\section{Effects of CANA on AGT Levels in Liver, Adipose}

Tissue, and Plasma in T2DM Mice

The major source of circulating AGT is the liver [20]. Recent studies have proposed that AGT produced by adipose tissue may also participate in regulation of RAS activity in adipose tissue and the circulation [21,22]. Accordingly, hepatic AGT mRNA, adipose tissue AGT mRNA, and plasma AGT protein levels were measured in this study. Expression levels of hepatic AGT mRNA were not different among all groups (online suppl. Fig. S5a). In adipose tissues of DM mice, AGT mRNA expression was lower than ND mice (online suppl. Fig. S5b). CANA treatment did not alter the expression levels in either ND or DM mice. Plasma AGT protein levels determined by ELISA demonstrated no differences among all groups (online suppl. Fig. S5c).

\section{Effects of CANA on Intrarenal AGT Expression in T2DM Mice}

As mentioned, renal proximal tubular AGT regulation has been shown to play crucial roles in the development of kidney injury and hypertension. Thus, renal cortical AGT mRNA and protein levels were evaluated. Renal cortical AGT mRNA levels in vehicle-treated DM mice were 2 -fold greater than in ND (Fig. 3a, $75 \pm 8$ copies/ng RNA in ND mice vs. $148 \pm 21$ copies/ng RNA in DM mice). Importantly, CANA treatment normalized the augmented renal cortical AGT mRNA levels in DM mice $(90 \pm 16$ copies/ng RNA). Furthermore, renal cortical AGT protein levels in DM mice were higher than in ND mice (Fig. 3b, $0.90 \pm 0.06$ in ND mice vs. $1.35 \pm 0.07$ in $\mathrm{DM}$ mice, AGT/ $\beta$-actin, arbitrary unit). Elevated AGT protein levels were not observed in DM mice treated with CANA $(0.92 \pm 0.08$ in CANA-treated DM mice, AGT/ $\beta$ actin, arbitrary unit), consistent with the mRNA results. In addition to renal cortical AGT levels, renin, ACE, AT1a, and SGLT2 mRNA levels in renal cortex were determined (online suppl. Table S1). Renin mRNA levels in CANA-treated DM mice were greater than ND mice. $\mathrm{ACE}$ and AT1a did not show any differences among the groups. SGLT2 mRNA levels in DM mice were higher than ND mice. This enhanced expression of SGLT2 mRNA was not observed in CANA-treated DM mice.

\section{Effects of CANA on Renal Oxidative Stress in T2DM Mice}

In vehicle-treated DM mice, urinary 8-isoprostane excretion rate gradually increased during the experimental period and reached $8.9 \pm 1.9 \mathrm{ng} /$ day on week 6 (Fig. 4). CANA treatment significantly lowered urinary 8-isoprostane levels in DM mice reaching $2.8 \pm 1.9 \mathrm{ng} /$ day on week 6 . The suppression of urinary 8 -isoprostane by CANA was rapid achieving $4.8 \pm 1.4 \mathrm{ng} /$ day in vehicletreated DM mice and $1.5 \pm 0.1 \mathrm{ng} /$ day in CANA-treated DM mice on week 1 . The suppressed urinary 8 -isoprostane excretion rates in the CANA-treated DM mice were sustained through week 6 . There was no difference in urinary 8-isoprostane excretion rate between vehicle and CANA-treated ND mice.

\section{Effects of CANA on Kidney Injury in T2DM Mice}

DM mice showed increased urinary albumin excretion rates even during week 1 of study (online suppl. Fig. S6a, $1137 \pm 333 \mu \mathrm{g} /$ day in vehicle-treated DM mice). In contrast, urinary albumin excretion rates were within the normal range in ND mice during the entire experimental period. CANA treatment did not attenuate the albuminuria in DM mice. Urinary protein levels showed similar trends in mice (online suppl. Fig. S6b). We also calculated the urinary albumin/creatinine and protein/creatinine ratios (online suppl. Fig. S6c, S6d), but these data also failed to provide any evidence that the albuminuria or proteinuria was improved during the period of treatment. 


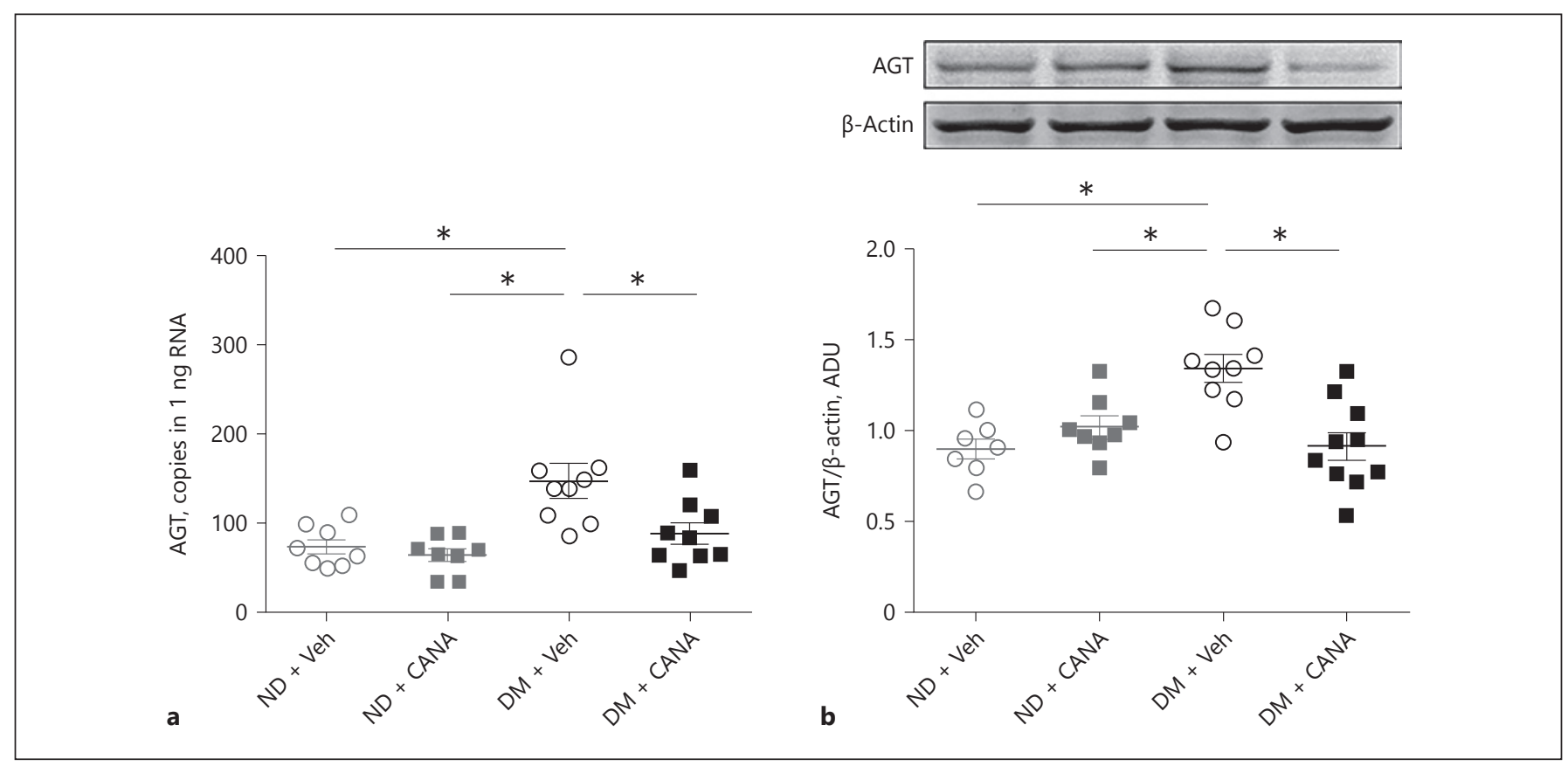

Fig. 3. Effects of CANA on intrarenal AGT expression in T2DM mice. $\mathbf{a}$ and $\mathbf{b}$ Intrarenal AGT mRNA and protein levels, respectively. ND + Veh: $n=8$, ND + CANA: $n=8$, DM + Veh: $n=9$, DM + CANA: $n=10$. Mean \pm SE. Asterisk $(p<0.05)$ indicates significant difference among groups.

Glomerular mesangial expansion was evaluated by PAS staining (Fig. 5a). Mesangial expansion scores in DM mice were slightly higher than in ND mice. However, the differences were not statistically significant. CANA treatment did not change the scores for mesangial expansion.

Accumulation of monocytes/macrophages, visualized as CD68-positive area, was increased in the interstitium of renal cortical tubules in vehicle-treated DM mice (Fig. 5b, brown staining indicated by arrows in the images), suggesting the progression of renal inflammation. As shown in Figure 5d, CANA treatment mitigated the monocyte/macrophage infiltration in DM mice.

Renal tubular fibrosis was observed only in vehicletreated DM mice (Fig. 5c, blue staining indicated by arrows in images). CANA-treated DM mice exhibited fibrotic scores similar to those in ND mice.

Proliferating cells, identified by PCNA staining, were observed mainly in renal cortical tubules in all groups (Fig. 5d, dark brown nuclei indicated by arrows in images). There was a statistically greater number of proliferating cells in the vehicle-treated DM mice compared to the ND mice. CANA treatment attenuated the increased cell proliferation seen in the DM mice.

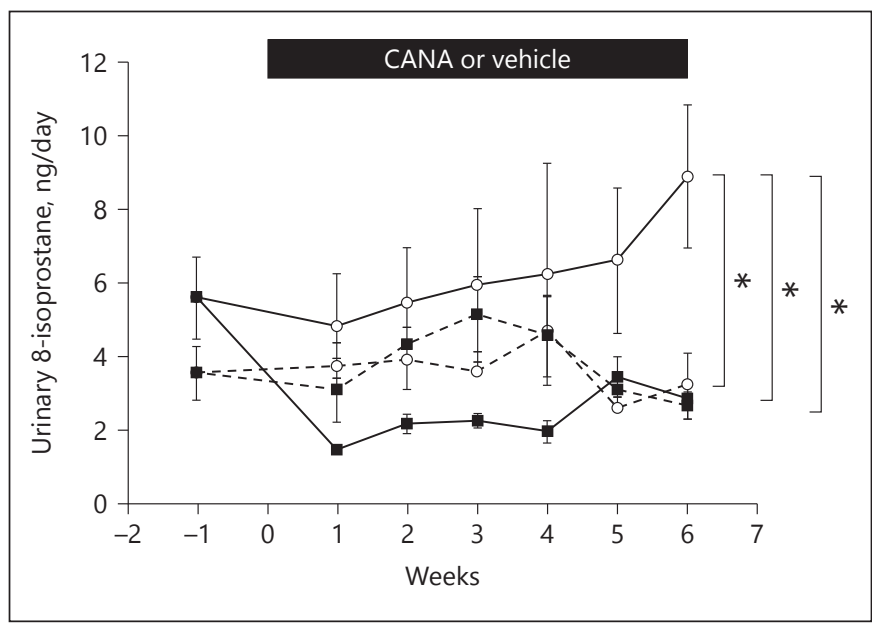

Fig. 4. Effects of CANA on renal oxidative stress in T2DM mice. ND + Veh: $n=4-8$, ND + CANA: $n=5-8$, DM + Veh: $n=5-8$, DM + CANA: $n=5-6$. Dotted lines show results in ND mice. Solid lines indicate results in DM mice. Open circles and closed squares are vehicle- and CANA-treated groups, respectively. Mean \pm SE. Asterisk $(p<0.05)$ indicates significant difference among groups. CANA, canagliflozin. 


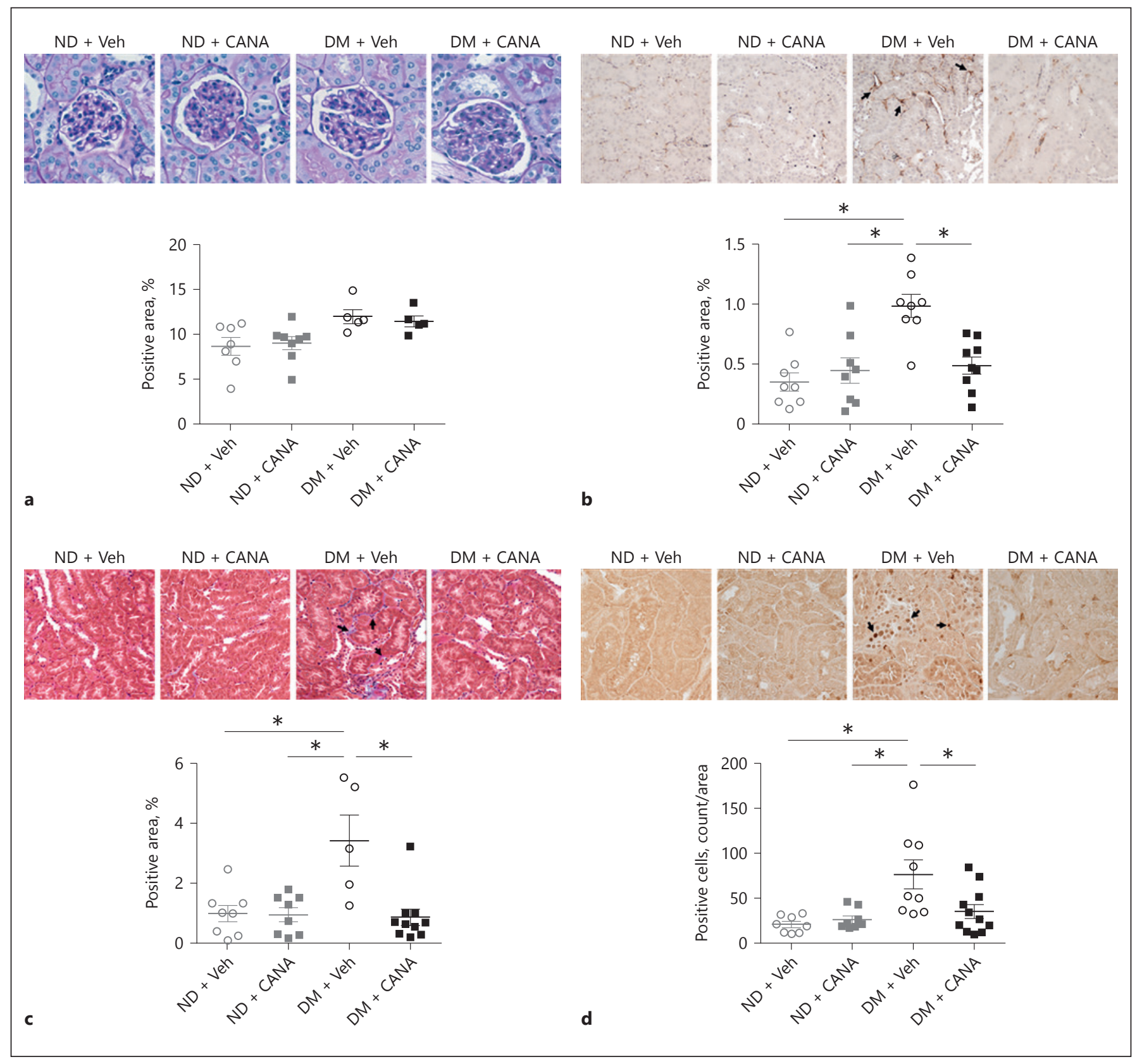

Fig. 5. Effects of CANA on kidney injury in T2DM mice. a Mesangial expansion. ND + Veh: $n=7, \mathrm{ND}+\mathrm{CANA}: n=8$, DM + Veh: $n=5$, DM + CANA: $n=5$. Mean \pm SE. There is no significant difference among groups. b Renal monocyte/macrophage infiltration. Brown color indicates filtered monocytes and macrophages in the images. ND + Veh: $n=8$, ND + CANA: $n=8$, DM + Veh: $n=8$, DM + CANA: $n=9$. Mean \pm SE. Asterisk $(p<0.05)$ indicates significant difference among groups. c Renal tubular fibrosis. Blue color indicates renal tubular fibrotic areas in the images. ND + Veh: $n=8$, ND + CANA: $n=8$, DM + Veh: $n=5$, DM + CANA: $n=10$. Mean \pm SE. Asterisk $(p<0.05)$ indicates significant difference among groups. d Renal cell proliferation. Brown color indicates nuclei of proliferating cells. ND + Veh: $n=8$, ND + CANA: $n=8, \mathrm{DM}+$ Veh: $n=10, \mathrm{DM}+$ CANA: $n=11$. Mean \pm SE. Asterisk $(p<0.05)$ indicates significant difference among groups. CANA, canagliflozin. 


\section{Discussion}

Our results demonstrate that CANA prevents intrarenal AGT upregulation and mitigates high blood pressure, renal tubular fibrosis, and renal inflammation in T2DM. These findings provide mechanistic explanations for the renal benefit of SGLT2 inhibitors observed in clinical trials.

Selective SGLT2 inhibitors have been developed to reduce glucose reabsorption in renal proximal tubules improving glycemic control in patients with T2DM [23]. As this has the effect of redistributing the glucose out of the PTC and into the lumen/urine, we undertook this study to determine the effect of this redistribution on intrarenal RAS activation, blood pressure, and kidney injury. In our animal model of diet-induced T2DM, CANA treatment was effective at normalizing blood glucose levels. Findings obtained from experiments using continuous glucose measurements demonstrated that CANA treatment rapidly reduced blood glucose levels within a few hours in DM mice, and the lowered blood glucose levels were sustained until the subsequent dose. Moreover, CANA treatment reduced the daily variation of blood glucose levels. These findings indicate that a single dose of CANA not only lowers blood glucose levels but also stabilizes the levels. In the DM mice, CANA treatment suppressed body weight gain similar to that reported in a large cohort analysis in patients with T2DM [24]. The effects of CANA on body weight gain were observed in the ND mice as well. This was accompanied with increased urinary glucose excretion rate in the ND mice without a change in blood glucose levels. This suggests that despite a modest reduction in body weight likely due to glycosuria, regulation of normal plasma glucose in ND CANA is likely due to hepatic gluconeogenesis.

In this study, NZO mice fed with HFD had SBP in the range of 130-140 $\mathrm{mm} \mathrm{Hg}$, which is consistent with previous findings in this strain [19]. Both tail-cuff and blood pressure telemetry systems demonstrated an antihypertensive effect of CANA administration in the DM mice. In contrast to the blood glucose responses, the amelioration of the increased SBP and MAP by CANA treatment occurred more gradually requiring more than 2 weeks of treatment before new levels were established. Therefore, acute blood glucose reduction by CANA is unlikely to directly reduce blood pressure in T2DM, suggesting that intermediary factors including RAS may be involved in the suppression of blood pressure caused by SGLT2 inhibition. Likewise, it is unlikely that the associated diuresis and naturesis directly contributed to the reductions in arterial pressure.

SGLT2 Inhibition Lowers Kidney AGT
Elevated intrarenal AGT has been shown to contribute to the progression of hypertension and kidney injury in many types of hypertension [3, 4]. In DM, renal proximal tubular AGT expression is stimulated by elevated glucose driving overproduction of ROS [8]. Thus, several studies have attempted to show suppressive effects of SGLT2 inhibitors on elevated intrarenal AGT in DM. Administration of Dapagliflozin, an SGLT2 inhibitor, in T2DM rats attenuated elevated urinary AGT excretion [25]. Furthermore, augmentation of AGT expression by high glucose and palmitic acid in cultured human PTC was blunted by Dapagliflozin [26]. In a small study, patients with T2DM, SGLT2 inhibitors exhibited a decreasing trend, but not significant, in urinary AGT excretion rates [27]. In contrast, the $\mathrm{db} / \mathrm{db}$ mouse model of T2DM exhibited decreased renal AGT mRNA expression that was enhanced by treatment with an SGLT2 inhibitor [28] and increased urinary AGT levels in response to Empagliflozin treatment of the OLETF DM rat [29] has been reported. Therefore, the effects of SGLT2 inhibition on intrarenal and/or urinary AGT augmentation in T2DM may be dependent on the animal model used. In this study, DM mice did not exhibit greater levels of circulating AGT and hepatic AGT production compared with the control mice, and there was no effect of CANA treatment. AGT mRNA levels in adipose tissue harvested from the diabetic mice were lower than in the control mice. Because mass of adipose tissue is increased in T2DM, the suppression of AGT levels in each adipocyte will be induced as a compensatory mechanism. Importantly, the increased expression of intrarenal AGT in the diabetic mice was attenuated by CANA treatment, which may contribute to the suppression of blood pressure by CANA in the DM mice. Since CANA treatment strongly inhibited intrarenal oxidative stress in the diabetic mice, the SGLT2 blockage may lead to attenuation of ROS generation and subsequent AGT augmentation in PTC. In contrast to the intrarenal AGT regulation, renin mRNA synthesis in renal cortex was enhanced by CANA in DM mice as shown in the Data Supplement. Recent studies have shown that juxtaglomerular cells and collecting duct cells strongly express renin in the kidneys. However, renin expression is regulated in different manners in these cells under disease conditions [3]. Thus, more detailed analyses in future studies will be required to delineate changes in renin expression by CANA.

Although studies including a clinical trial have demonstrated that CANA treatments improve glomerular function [12] and potentially slow progression of albuminuria [13], in this study, CANA treatment did not improve glomerular mesangial expansion and albuminuria in DM 
mice. Other studies also showed no changes in albuminuria by SGLT2 inhibitors [27, 30]. The issue is complicated in that the duration of treatment appears to influence the effects of treatment on albuminuria. Vallon et al. [31] reported attenuation of the albuminuria after 15 weeks of empagliflozin to diabetic Akita mice. Terami et al. [32] showed that treatment with dapagliflozin prevented the development of albuminuria in $\mathrm{db} / \mathrm{db}$ mice, but they did not test the effects on the mice that already developed albuminuria. Wang et al. [28] showed attenuation of the albuminuria in the $\mathrm{db} / \mathrm{db}$ mice after 12 weeks of treatment. Nevertheless, in our diabetic model, CANA treatment attenuated the progression of monocyte/macrophage infiltration into the interstitium of renal cortical tubules, renal cortical tubular fibrosis, and renal cortical cell proliferation. Thus, mitigation of glomerular dysfunction by CANA treatment may require a longer period than it takes for CANA to attenuate renal tubular fibrosis, inflammation, and cell proliferation. Indeed, in clinical trials, the mean GFR initially falls slightly followed by stabilization and then improvement. Thus, the short-term effect is compatible with our data.

In this study, CANA administration was started shortly after onset of diabetes, thus providing clear data on the effects on SGLT2 inhibition in the absence of confounding factors. This yields a mechanism for CANA treatment to slow or prevent the development of diabetic nephropathy. Additional studies are needed to examine the ability of CANA administration to slow the progression of renal disease in the clinical scenario where the duration of diabetes is greater and the patient has received other antihyperglycemic therapies. Likewise, these mice did not receive any antihypertensive therapy, whereas in the clinical setting, diabetic patients are commonly prescribed an angiotensinconverting enzyme inhibitor or angiotensin receptor blocker. Some studies demonstrate a further reduction in blood pressure and kidney injury with the combination of SGLT2 inhibitors and ACE inhibitors or AT1 receptor blockers in DM models following induction of hyperglycemia [33]. Clinically, SGLT2 inhibition has been shown to have an additive effect on blood pressure lowering in the patients receiving ACE inhibitors or AT1 receptor blockers $[34,35]$. While the additional blood pressure lowering may be due RAS-independent effects, it may also result from incomplete RAS blockade, especially in the proximal tubules. Thus, additional studies examining the combination of SGLT2 inhibition with angiotensin-converting enzyme inhibition in models of type 2 diabetes are warranted.

In conclusion, this study demonstrated that SGLT2 inhibition by CANA suppresses intrarenal oxidative stress and AGT upregulation, and this is associated with mitiga- tion of the hypertension and the kidney injury including renal tubular fibrosis and inflammation that occurs in T2DM. Accordingly, this study elucidates mechanisms underlying beneficial effects of SGLT2 inhibitions on the development of hypertension and kidney injury in T2DM.

\section{Acknowledgments}

The authors thank Daniel J. Lightell, Jr. and Alexander Castillo (Tulane University) for their excellent technical assistance.

\section{Statement of Ethics}

Animal experiments were approved by the Tulane University Institutional Animal Care and Use Committee and conform to the guidelines of the National Institutes of Health on the care and use of laboratory animals (Protocol \#4389).

\section{Disclosure Statement}

This study was supported by a grant to L.G.N. from Janssen Pharmaceuticals, Inc.

\section{Funding Sources}

This study was supported by Janssen (28431754DIA4010,/ L.G.N.), the NIGMS IDeA Program (COBRE, P30GM103 337/L.G.N.), the NHLBI (R01HL127092/Woods), and the NIDDK (R01DK107694/Satou).

\section{Author Contributions}

L.G.N., T.C.W., V.A.F., and R.S. obtained the funding for this study and were involved in the conception and design, data interpretation, and critical review for this manuscript. In addition, K.M., A.K., C.M.D., and N.C.K. were involved in the data collection, data interpretation, and manuscript drafting. All authors read and approved the final manuscript and figures.

References

Woods/Satou/Miyata/Katsurada/Dugas/ Klingenberg/Fonseca/Navar
1 Elmarakby AA, Abdelsayed R, Yao Liu J, Mozaffari MS. Inflammatory cytokines as predictive markers for early detection and progression of diabetic nephropathy. EPMA J. 2010 Mar;1(1):117-29.

2 Varga ZV, Giricz Z, Liaudet L, Haskó G, Ferdinandy P, Pacher P. Interplay of oxidative, nitrosative/nitrative stress, inflammation, cell death and autophagy in diabetic cardiomyopathy. Biochim Biophys Acta. 2015 Feb; 1852(2):232-42. 
3 Navar LG, Prieto MC, Satou R, Kobori H. Intrarenal angiotensin II and its contribution to the genesis of chronic hypertension. Curr Opin Pharmacol. 2011 Apr;11(2):180-6.

4 Kobori H, Nangaku M, Navar LG, Nishiyama A. The intrarenal renin-angiotensin system: from physiology to the pathobiology of hypertension and kidney disease. Pharmacol Rev. 2007 Sep;59(3):251-87.

5 Miyata K, Ohashi N, Suzaki Y, Katsurada A, Kobori H. Sequential activation of the reactive oxygen species/angiotensinogen/reninangiotensin system axis in renal injury of type 2 diabetic rats. Clin Exp Pharmacol Physiol. 2008 Aug;35(8):922-7.

6 Park S, Bivona BJ, Kobori H, Seth DM, Chappell MC, Lazartigues E, et al. Major role for ACE-independent intrarenal ANG II formation in type II diabetes. Am J Physiol Renal Physiol. 2010 Jan;298(1):F37-48.

7 Kamiyama M, Zsombok A, Kobori H. Urinary angiotensinogen as a novel early biomarker of intrarenal renin-angiotensin system activation in experimental type 1 diabetes. J Pharmacol Sci. 2012;119(4):314-23.

8 Hsieh TJ, Fustier P, Wei CC, Zhang SL, Filep JG, Tang SS, et al. Reactive oxygen species blockade and action of insulin on expression of angiotensinogen gene in proximal tubular cells. J Endocrinol. 2004 Dec;183(3):535-50.

9 List JF, Woo V, Morales E, Tang W, Fiedorek FT. Sodium-glucose cotransport inhibition with dapagliflozin in type 2 diabetes. Diabetes Care. 2009 Apr;32(4):650-7.

10 Foote C, Perkovic V, Neal B. Effects of SGLT2 inhibitors on cardiovascular outcomes. Diab Vasc Dis Res. 2012 Apr;9(2):117-23.

11 Weber MA, Mansfield TA, Cain VA, Iqbal N, Parikh S, Ptaszynska A. Blood pressure and glycaemic effects of dapagliflozin versus placebo in patients with type 2 diabetes on combination antihypertensive therapy: a randomised, double-blind, placebo-controlled, phase 3 study. Lancet Diabetes Endocrinol. 2016 Mar;4(3):211-20.

12 Yale JF, Bakris G, Cariou B, Yue D, DavidNeto E, Xi L, et al. Efficacy and safety of canagliflozin in subjects with type 2 diabetes and chronic kidney disease. Diabetes Obes Metab. 2013 May; 15(5):463-73.

13 Neal B, Perkovic V, Mahaffey KW, de Zeeuw D, Fulcher G, Erondu N, et al.; CANVAS Program Collaborative Group. Canagliflozin and Cardiovascular and Renal Events in Type 2 Diabetes. N Engl J Med. 2017 Aug;377(7): 644-57.

14 Bielschowsky F, Bielschowsky M. The New Zealand strain of obese mice; their response to stilboestrol and to insulin. Aust J Exp Biol Med Sci. 1956 Jun;34(3):181-98.

15 Mirhashemi F, Scherneck S, Kluth O, Kaiser $\mathrm{D}$, Vogel H, Kluge R, et al. Diet dependence of diabetes in the New Zealand Obese (NZO) mouse: total fat, but not fat quality or sucrose accelerates and aggravates diabetes. Exp Clin Endocrinol Diabetes. 2011 Mar;119(3):16771.

16 Liu J, Edgington-Giordano F, Dugas C, Abrams A, Katakam P, Satou R, et al. Regulation of Nephron Progenitor Cell Self-Renewal by Intermediary Metabolism. J Am Soc Nephrol. 2017 Nov;28(11):3323-35.

17 O'Leary R, Penrose H, Miyata K, Satou R. Macrophage-derived IL-6 contributes to ANG II-mediated angiotensinogen stimulation in renal proximal tubular cells. Am J Physiol Renal Physiol. 2016 May; 310(10):F1000-7

18 Satou R, Kobori H, Katsurada A, Miyata K, Navar LG. Quantification of intact plasma AGT consisting of oxidized and reduced conformations using a modified ELISA. Am J Physiol Renal Physiol. 2016 Dec; 311(6):F1211-6.

19 Tsukahara C, Sugiyama F, Paigen B, Kunita S, Yagami K. Blood pressure in 15 inbred mouse strains and its lack of relation with obesity and insulin resistance in the progeny of an $\mathrm{NZO} /$ HILtJ x $\mathrm{C} 3 \mathrm{H} / \mathrm{HeJ}$ intercross. Mamm Genome. 2004 Dec;15(12):943-50.

20 Brasier AR, Li J. Mechanisms for inducible control of angiotensinogen gene transcription. Hypertension. 1996 Mar;27(3 Pt 2):46575.

21 Pahlavani M, Kalupahana NS, Ramalingam L, Moustaid-Moussa N. Regulation and Functions of the Renin-Angiotensin System in White and Brown Adipose Tissue. Compr Physiol. 2017 Sep;7(4):1137-50.

22 Yiannikouris F, Gupte M, Putnam K, Thatcher S, Charnigo R, Rateri DL, et al. Adipocyte deficiency of angiotensinogen prevents obesity-induced hypertension in male mice. Hypertension. 2012 Dec;60(6):1524-30.

23 van Bommel EJ, Muskiet MH, Tonneijck L, Kramer MH, Nieuwdorp M, van Raalte DH. SGLT2 Inhibition in the Diabetic KidneyFrom Mechanisms to Clinical Outcome. Clin J Am Soc Nephrol. 2017 Apr;12(4):700-10.

24 Cefalu WT, Stenlöf K, Leiter LA, Wilding JP, Blonde L, Polidori D, et al. Effects of canagliflozin on body weight and relationship to HbAlc and blood pressure changes in patients with type 2 diabetes. Diabetologia. 2015 Jun;58(6):1183-7.

25 Shin SJ, Chung S, Kim SJ, Lee EM, Yoo YH, Kim JW, et al. Effect of Sodium-Glucose CoTransporter 2 Inhibitor, Dapagliflozin, on Renal Renin-Angiotensin System in an Animal Model of Type 2 Diabetes. PLoS One. 2016 Nov;11(11):e0165703.

26 Han E, Shin E, Kim G, Lee JY, Lee YH, Lee $\mathrm{BW}$, et al. Combining SGLT2 Inhibition With a Thiazolidinedione Additively Attenuate the Very Early Phase of Diabetic Nephropathy Progression in Type 2 Diabetes
Mellitus. Front Endocrinol (Lausanne). 2018 Jul;9:412.

27 Yoshimoto T, Furuki T, Kobori H, Miyakawa M, Imachi H, Murao K, Nishiyama A. Effects of sodium-glucose cotransporter 2 inhibitors on urinary excretion of intact and total angiotensinogen in patients with type 2 diabetes. J Investig Med. 2017 Oct;65(7):1057-1061.

28 Wang XX, Levi J, Luo Y, Myakala K, HermanEdelstein M, Qiu L, et al. SGLT2 Protein Expression Is Increased in Human Diabetic Nephropathy: SGLT2 PROTEIN INHIBITION DECREASES RENAL LIPID ACCUMULATION, INFLAMMATION, AND THE DEVELOPMENT OF NEPHROPATHY IN DIABETIC MICE. J Biol Chem. 2017 Mar; 292(13):5335-48

29 Takeshige Y, Fujisawa Y, Rahman A, Kittikulsuth W, Nakano D, Mori H, et al. A sodium-glucose co-transporter 2 inhibitor empagliflozin prevents abnormality of circadian rhythm of blood pressure in salt-treated obese rats. Hypertens Res. 2016 Jun;39(6): 415-22.

30 Gallo LA, Ward MS, Fotheringham AK, Zhuang A, Borg DJ, Flemming NB, et al. Once daily administration of the SGLT2 inhibitor, empagliflozin, attenuates markers of renal fibrosis without improving albuminuria in diabetic db/db mice. Sci Rep. 2016 May;6(1): 26428.

31 Vallon V, Gerasimova M, Rose MA, Masuda T, Satriano J, Mayoux E, et al. SGLT2 inhibitor empagliflozin reduces renal growth and albuminuria in proportion to hyperglycemia and prevents glomerular hyperfiltration in diabetic Akita mice. Am J Physiol Renal Physiol. 2014 Jan;306(2):F194-204.

32 Terami N, Ogawa D, Tachibana H, Hatanaka T, Wada J, Nakatsuka A, et al. Long-term treatment with the sodium glucose cotransporter 2 inhibitor, dapagliflozin, ameliorates glucose homeostasis and diabetic nephropathy in $\mathrm{db} / \mathrm{db}$ mice. PLoS One. 2014 Jun; 9(6):e100777

33 Kojima N, Williams JM, Slaughter TN, Kato S, Takahashi T, Miyata N, et al. Renoprotective effects of combined SGLT2 and ACE inhibitor therapy in diabetic Dahl S rats. Physiol Rep. 2015 Jul;3(7):e12436.

34 Mancia G, Cannon CP, Tikkanen I, Zeller C, Ley L, Woerle HJ, et al. Impact of Empagliflozin on Blood Pressure in Patients With Type 2 Diabetes Mellitus and Hypertension by Background Antihypertensive Medication. Hypertension. 2016 Dec;68(6):1355-64.

35 Kario K, Okada K, Kato M, Nishizawa M, Yoshida T, Asano T, et al. 24-Hour Blood Pressure-Lowering Effect of an SGLT-2 Inhibitor in Patients with Diabetes and Uncontrolled Nocturnal Hypertension: Results from the Randomized, Placebo-Controlled SACRA Study. Circulation. 2018 Nov 29. 